\title{
HADRONIC RESONANCE PRODUCTION WITH THE ALICE EXPERIMENT IN pp AND Pb-Pb COLLISIONS AT LHC ENERGIES
}

\author{
Sergey Kiselev ${ }^{a}$ for the ALICE collaboration \\ Institute for Theoretical and Experimental Physics, 117259 Moscow, Russia \\ Abstract. Hadronic resonances $\mathrm{K}^{*}(892)^{0}, \phi(1020)$ and $\Sigma(1385)^{ \pm}$have been mea- \\ sured by the ALICE experiment in pp collisions at $\sqrt{s}=7 \mathrm{TeV}$ and in $\mathrm{Pb}-\mathrm{Pb}$ \\ collisions at $\sqrt{s_{\mathrm{NN}}}=2.76 \mathrm{TeV}$. Transverse momentum spectra, particle ratios, nu- \\ clear modification factor and comparison with model predictions are discussed. In \\ addition, ALICE results are compared with data obtained at RHIC energy.
}

Resonance production plays an important role both in elementary and in heavy-ion collisions. In pp collisions, it provides a reference for nuclear collisions and also data for tuning event generators inspired by Quantum Chromodynamics. In heavy-ion collisions, the in-medium effects related to the highdensity and/or high temperature of the medium can modify the properties of short-lived resonances such as their masses, widths, and even their spectral shapes [1]. Moreover, due to short life time the regeneration and rescattering effects become important and can be used to estimate the timescale between chemical and kinetic freeze-out [2].

The resonances have been identified via their main hadronic decay channels: $\mathrm{K}^{*}(892)^{0} \rightarrow \pi^{ \pm}+\mathrm{K}^{\mp}, \phi(1020) \rightarrow \mathrm{K}^{+}+\mathrm{K}^{-}, \Sigma(1385)^{ \pm} \rightarrow \Lambda+\pi^{ \pm}$. The analysis of the $\mathrm{K}^{*}(892)^{0}$ and $\phi(1020)$ mesons in pp collisions at $\sqrt{s}=7 \mathrm{TeV}$ was described in detail in 3]. Transverse momentum spectra of the $\mathrm{K}^{*}$ and $\phi$ mesons have been compared to a number of PYTHIA 44 tunes and the PHOJET [5] event generators. None of them is capable of providing a fully satisfactory description of the data. Figure 1 shows comparison of $\Sigma(1385)$ spectra with event generator predictions. The models underpredict the data. The $\mathrm{K}^{*} / \mathrm{K}^{-}$and $\phi / \mathrm{K}^{-} \mathrm{dN} / \mathrm{dy}$ ratios are found to be independent of energy up to $7 \mathrm{TeV}$ 3]. Comparing to results from RHIC top energy, $\Sigma^{*} / \pi^{-}$and $\Sigma^{*} / \mathrm{K}^{-}$ratios are also independent of energy within errors and agree with the thermal model [6] predictions at $\sqrt{s}=7 \mathrm{TeV}$. For the $\Sigma^{*} / \Xi^{-}$ratio there is a hint of decrease with energy and its value at $\sqrt{s}=7 \mathrm{TeV}$ is overpredicted by the thermal model [7].

The mesonic resonances $\mathrm{K}^{*}$ and $\phi$ have been also measured in $\mathrm{Pb}-\mathrm{Pb}$ collisions at $\sqrt{s_{\mathrm{NN}}}=2.76 \mathrm{TeV}$. For $\mathrm{K}^{*}(\phi)$ the $\left\langle p_{\mathrm{T}}\right\rangle$ at LHC energy is $\sim 20 \%(\sim 30 \%)$ higher than that observed at RHIC energy [8]. A weak centrality dependence was observed in the $\mathrm{K}^{*} / \mathrm{K}^{-}$ratio, while the $\phi / \mathrm{K}^{-}$ratio is independent of the collision centrality [9]. The decreasing trend in the $\mathrm{K}^{*} / \mathrm{K}^{-}$ratio suggests a possible increase of hadronic rescattering in the most central collisions. The nuclear modification factor $R_{\mathrm{AA}}$ for four different particle species is shown in Figure2 For $p_{\mathrm{T}}<2.5 \mathrm{GeV} / c$, the $\phi R_{\mathrm{AA}}$ appears to follow $R_{\mathrm{AA}}$ of $\mathrm{p}$, while for

${ }^{a}$ E-mail: Sergey.Kiselev@cern.ch 


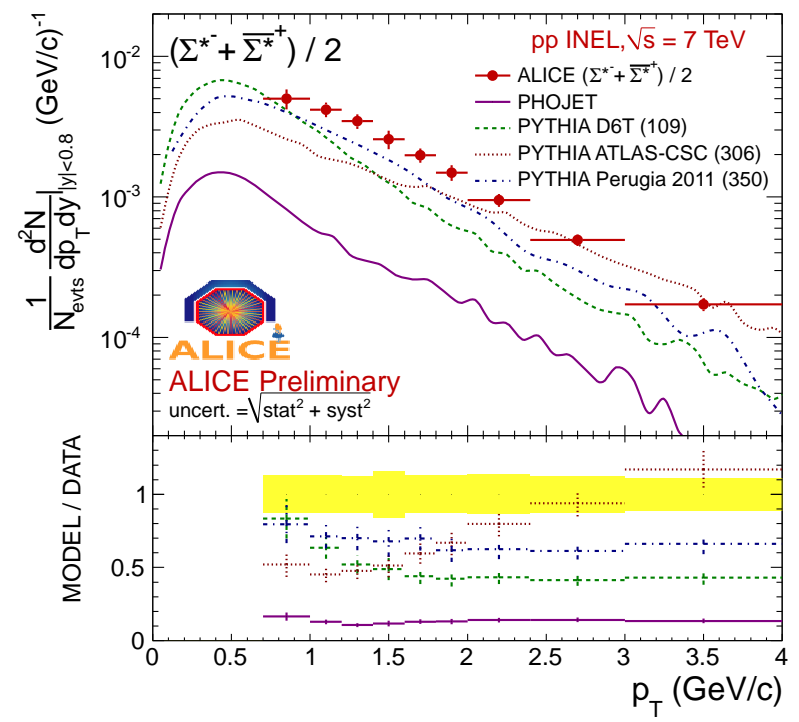

Figure 1: Comparison of transverse momentum spectra of $\Sigma^{*}$ in pp collisions at $\sqrt{s}=7 \mathrm{TeV}$ with PHOJET and PYTHIA tunes D6T, ATLAS-CSC and Perugia 2011.

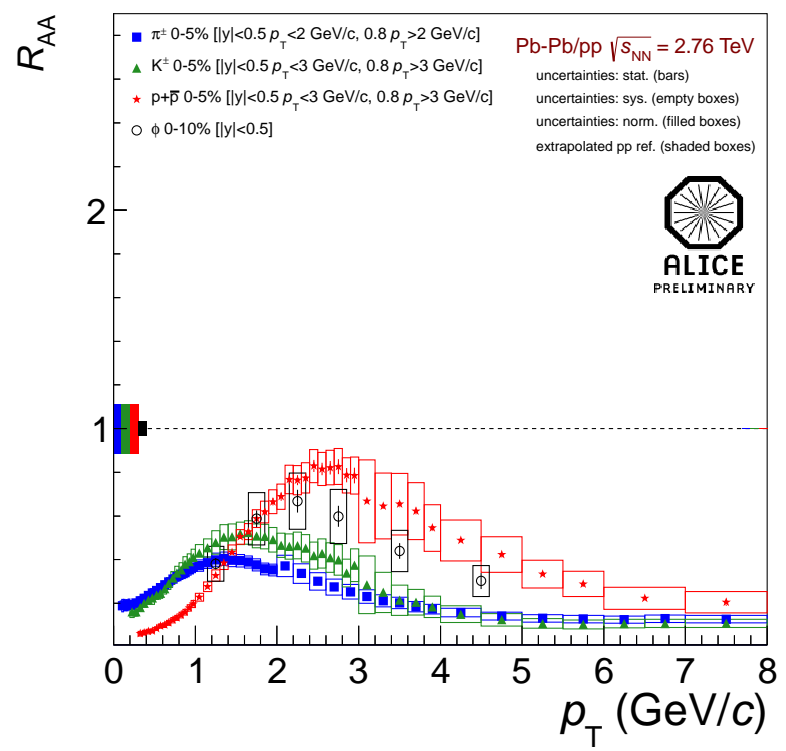

Figure 2: $R_{\mathrm{AA}}$ of various particles. 
mid-to-high $p_{\mathrm{T}}$, the $\phi R_{\mathrm{AA}}$ tends to be between $R_{\mathrm{AA}}$ of the mesons ( $\pi$ and $\mathrm{K}$ ) and $\mathrm{p}$. The $\phi / p$ ratio as a function of $p_{\mathrm{T}}$ demonstrate a flat shape for central collisions, Figure 3. That indicates that the differences in shape between the

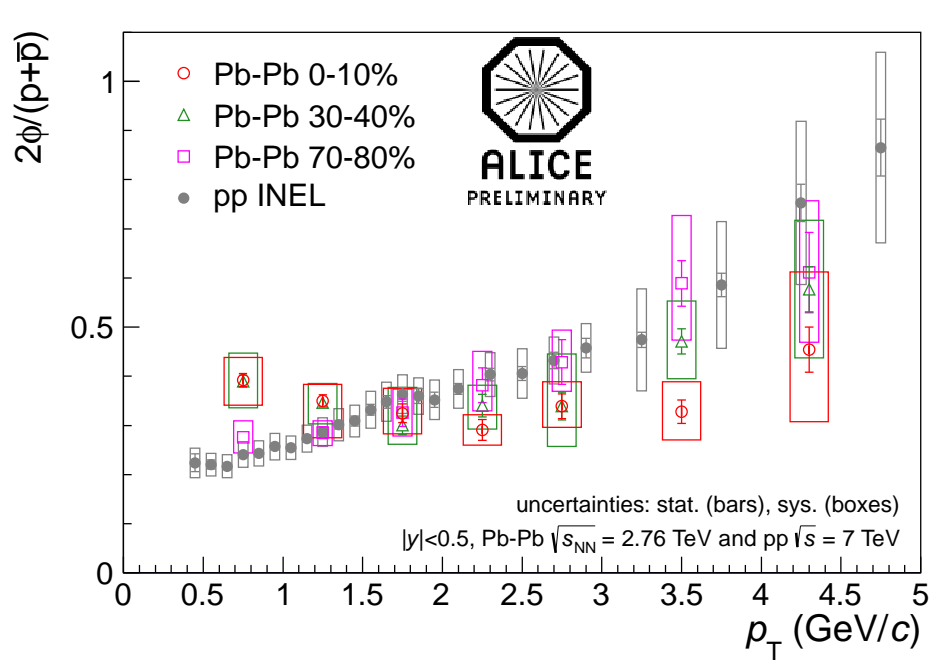

Figure 3: $\phi / p$ ratio.

$\phi$ and $\mathrm{p} R_{\mathrm{AA}}$ are most likely due to the pp reference. The ratio for peripheral collisions is similar to pp at $\sqrt{s}=7 \mathrm{TeV}$.

[1] G. E. Brown and M. Rho, Phys. Rev. Lett. 66, 2720 (1991). R. Rapp, Nucl. Phys. A 725, 254 (2003). E. V. Shuryak and G. Brown, Nucl. Phys. A 717, 322 (2003).

[2] G. Torrieri and J. Rafelski, Phys. Lett. B 509, 239 (2001).

[3] B. Abelev et al., (ALICE Collaboration), Eur. Phys. J. C 72, 2183 (2012).

[4] T. Sjöstrand, S. Mrenna and P. Skands, J. High Energy Phys. 05, 026 (2006).

[5] R. Engel et al., Z. Phys. C 66, 203 (1995); R. Engel and J. Ranft, Phys. Rev. D 54, 4144 (1996).

[6] F. Becattini et al., J. Phys. G 38, 025002 (2011).

[7] S. Kiselev, for the ALICE Collaboration, PoS(Baldin ISHEPP XXI)054 (2012).

[8] A. G. Knospe, for the ALICE Collaboration, J. Phys.: Conf. Series 410 012018 (2013), arXiv:1301.1644 [nucl-ex].

[9] S. Singha, for the ALICE Collaboration, PoS(CPOD 2013)055 (2013). 